Marquette University

e-Publications@Marquette

Physics Faculty Research and Publications

Physics, Department of

9-1-2002

\title{
Normal Mode Mixing and Ferromagnetic Resonance Linewidth
}

Andrew Kunz

Marquette University, andrew.kunz@marquette.edu

R. D. McMichael

National Institute of Standards \& Technology

Accepted version. IEEE Transactions on Magnetics, Vol. 38, No. 5 (September 2002): 2400-2402. DOI. (C) 2002 IEEE. Used with permission.

Andrew Kunz was affiliated with the National Institute of Standards and Technology at the time of publication. 


\title{
Marquette University
}

\section{e-Publications@Marquette}

\section{Physics Faculty Research and Publications/College of Arts and Sciences}

This paper is NOT THE PUBLISHED VERSION; but the author's final, peer-reviewed manuscript. The published version may be accessed by following the link in the citation below.

IEEE Transactions on Magnetics, Vol. 38, No. 5 (December 10, 2002): 2400-2402. DOI. This article is (C) Institute of Electrical and Electronics Engineers (IEEE) and permission has been granted for this version to appear in e-Publications@Marquette. IEEE does not grant permission for this article to be further copied/distributed or hosted elsewhere without the express permission from IEEE.

\section{Normal Mode Mixing and Ferromagnetic Resonance Linewidth}

\author{
A. Kunz \\ Nat. Inst. of Stand. \& Technol., Gaithersburg, MD \\ R.D. McMichael \\ Nat. Inst. of Stand. \& Technol., Gaithersburg, MD
}

\section{IEEE Keywords}

Magnetic resonance, Couplings, Transistors, Magnetic anisotropy, Perpendicular magnetic anisotropy, Magnetostatic waves, Magnetic materials, NIST, Scattering, Magnetic films

\begin{abstract}
:
The normal modes of an inhomogeneous thin film are obtained by diagonalization of the perturbed Hamiltonian. The resulting modes are mixtures of the spin-wave modes and the uniform mode. We find that the ferromagnetic resonance intensity spectrum of the diagonalized system has a Lorentzian profile, and that the results correspond to the two-magnon model for weak perturbations. For stronger perturbations, the density of states is smoothed, and the spectrum becomes asymmetric due to the
\end{abstract}


low-frequency cutoff of the spin-wave manifold. The technique is expected to be valid for perturbation amplitudes that are large enough to invalidate the assumptions of the two-magnon model.

SECTION I.

\section{Introduction}

In The ferromagnetic resonance of polycrystalline bulk materials, the effects of inhomogeneity can be classified into the strong coupling limit, where the magnetostatic coupling between grains is much greater than the anisotropy field, $M_{\mathrm{s}} \gg H_{\mathrm{a}}$, and the weak coupling limit where $M_{\mathrm{s}} \ll H_{\mathrm{a}}{ }^{[1]}$. A good description of the strong coupling limit is available from the two-magnon model of ferromagnetic resonance linewidth ${ }^{[2][3]-[4][5][6][7]}$ in which the magnetostatic and exchange interactions are taken into account via the spin-wave dispersion relation for the material, and the inhomogeneities are treated as perturbations. In the weak coupling limit, the resonance spectrum can be approximated by the superposition of resonances from noninteracting grains. Intermediate coupling conditions in bulk materials have been approached using a self-consistency approach ${ }^{[8]}$ to calculate higher order effects, and by considering direct perturbations of the spin-wave manifold ${ }^{[9]}$.

Intermediate coupling conditions may be more prevalent in thin films since the magnetostatic interactions can be much weaker than in the bulk, so that for a large value of $H_{\mathrm{a}}$, the approximations involved in a two-magnon model of linewidth may not be valid. It would be useful, therefore, to extend our understanding of thin films ferromagnetic resonance out of the strong coupling limit where inhomogeneity is treated as a perturbation and into the intermediate coupling regime.

The dynamics of thin uniform films can be described by a set of normal modes, known under various approximations as Damon-Eshbach [10], magnetoexchange [11], [12], or spin-wave modes. The spin-wave modes are labeled by the wave vector $\boldsymbol{k}$, with $\boldsymbol{k}=0$ representing the uniform precession mode that couples to the driving microwave field. When nonuniformities are introduced, the plane-wave states still form an orthogonal basis, but they are no longer the eigenstates of the Hamiltonian. In particular, the uniform precession that is driven in FMR experiments is not an eigenmode of the perturbed film. The inclusion of nonuniformities couples the $\boldsymbol{k}=0$ uniform mode to other, $\boldsymbol{k} \neq 0$ nonuniform modes of the film. The fully perturbed Hamiltonian is written as

$$
\begin{gathered}
H=\hbar \omega_{0} a_{0}^{\dagger} a_{0}+\sum_{k \neq 0} \hbar \omega_{\mathbf{k}} a_{\mathbf{k}}^{\dagger} a_{\mathbf{k}}+\sum_{\mathbf{k}^{\prime} \neq \mathbf{k}} \\
\cdot\left(A_{\mathbf{k}^{\prime} \mathbf{k}} a_{\mathbf{k}^{\prime}} a_{\mathbf{k}}^{\dagger}+A_{\mathbf{k}^{\prime} \mathbf{k}}^{*} a_{\mathbf{k}^{\prime}}^{\dagger} a_{\mathbf{k}}\right)
\end{gathered}
$$

where $a_{\mathbf{k}}$ and $a_{\mathbf{k}}^{\dagger}$ are the lowering and raising operators for the spin waves modes for the uniform film. The second sum includes the uniform spin-wave mode, and $A_{\mathbf{k}^{\prime} \mathbf{k}}$ gives the strength of the inhomogeneity which couples the various spin-wave modes.

For small $A_{0 \mathbf{k}}$ the FMR linewidth is described by the two-magnon model of FMR damping. This theory treats inhomogeneities as perturbations giving a scattering rate that is found by Fermi's Golden Rule 
(FGR), and that is observed experimentally by the broadening of the ferromagnetic resonance peak. The rate given for the scattering of $\mathbf{k}^{\prime}=0$ to $\mathbf{k} \neq 0$ magnons is

$$
\Gamma_{0, \mathbf{k}}=\frac{2 \pi}{\hbar} \sum_{\mathbf{k}}\left|A_{0 \mathbf{k}}\right|^{2} \delta\left(\hbar \omega_{0}-\hbar \omega_{\mathbf{k}}\right)
$$

where only modes coupled by $A_{0 \mathbf{k}}$ with the same energy contribute to the total overall scattering. Because $(2)$ is a result of perturbation theory, the validity of this approximation is limited to values of $A_{0 \mathbf{k}}$ that are in some sense small. For cases where $\left\langle A_{0 \mathbf{k}}^{2}\right\rangle$ is independent of $\boldsymbol{k}$, then $\delta$-function is (2) effectively measures the density of states at $\omega_{0}=\omega_{\mathbf{k}}$.

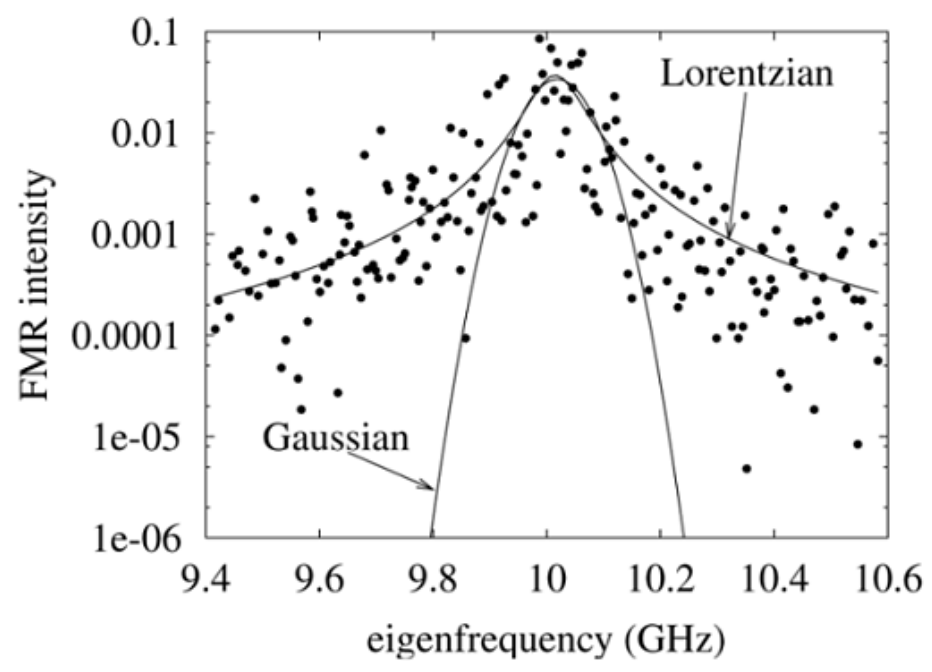

Fig. 1. Best fit for the intensity of spin-wave modes contributing to the FMR linewidth. The tails of the data are best described by a Lorenzian function.

In this paper, we take an approach valid for large perturbations by rediagonalizing the full Hamiltonian by including interactions between all spin-wave modes available for the film. The diagonalization approach provides insight into more complicated cases in which the spin-wave density of states is not uniform. 


\section{SECTION II.}

\section{Rediagonalization}

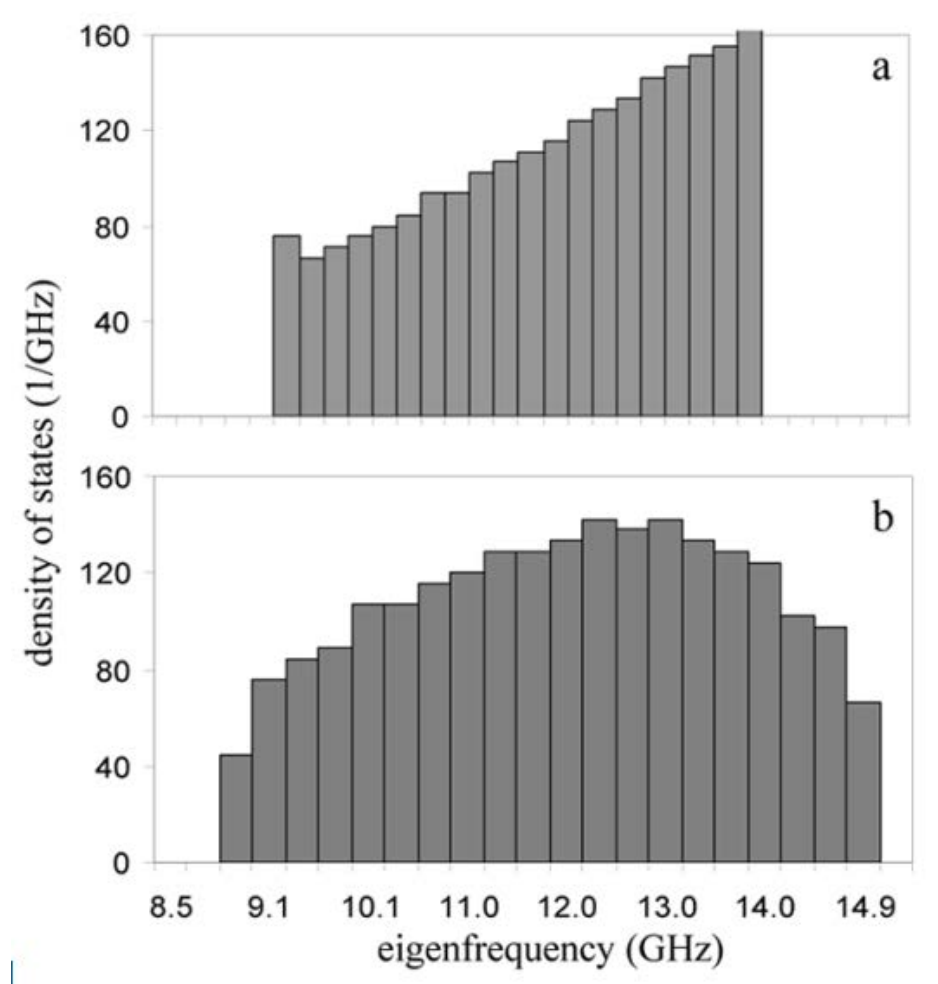

Fig. 2. Density of states profile for (a) 20-nm-thick uniform Permalloy film and (b) for a strongly perturbed films ( $A \mathrm{rms}=0.05 \mathrm{GHz}$ ). As the strength of the perturbation increases, the density of states becomes more evenly distributed.

The Hamiltonian (1) consists of a series of spin-wave modes for the uniform film and a perturbation. In matrix representation the perturbation can be rewritten as

$$
H^{\prime}=a^{\dagger} A a^{(3)}
$$

where $A$ is an $N \times N$ matrix and $a$ is an $N$-vector containing the annihilation vectors $a_{\mathbf{k}}$. By including the eigenfrequencies, $\omega_{\mathbf{k}}$, of the uniform film along the diagonal, the matrix representation of (1) is recovered. Diagonalization of this Hamiltonian allows one to pick out the new eigenvalues and normal modes of the perturbed system. To do so, we introduce a unitary matrix $U$. In doing so the Hamiltonian takes the form

$$
H=a^{\dagger} U\left(U^{-1} A U\right) U^{-1} a(4)
$$


where $U^{-1} A U$ is diagonal and identically the new eigenvalue spectrum for the perturbed system. The new basis $\left(U^{-1} a\right)$ consists of a new set of eigenmodes containing linear combinations of the original uniform film spin-wave modes. To implement the procedure, the perturbation matrix, $A$, is composed from a Gaussian distribution of random complex numbers, $A_{\mathbf{k k}^{\prime}}$, centered about zero with varying strength characterized by the root-mean-square ( $\mathrm{rms}$ ) value. The other tunable parameter in the numerical calculations is the density of states in the unperturbed system, most easily changed by increasing the number of modes available to couple to within a fixed frequency range. In this paper, we present results for both uniform and nonuniform densities of spin-wave states.

\section{SECTION III.}

\section{Results}

Starting with a uniform distribution of spin-wave states, we find that diagonalization produces a symmetric distribution of new eigenstates about the frequency of interest. The eigenstates of the perturbed Hamiltonian are mixtures of all the original spin-wave modes including the uniform mode, and we compute the FMR intensity spectrum by taking the amplitude of the uniform mode in each eigenmode, $i, U_{i 0}$, and plotting it versus the eigenfrequency, see Fig. 1. In an unperturbed film, the plot would consist of a single spike at $10 \mathrm{GHz}$.

One might have expected that the addition of random perturbations would have produced a Gaussian lineshape, but we find that least squares fits to the intensity yield a better fit to a Lorenzian, especially in the tails of the spectrum away from resonance. The two-magnon model (2) also predicts a Lorenzian lineshape by virtue of the fact that it gives a damping rate.

In a series of runs with different initial densities of states and small values of $A_{0 \mathbf{k}}$, we found that the spectrum width increases linearly with the initial density of states, and roughly quadratically with the perturbation amplitude, consistent with (2).

We also calculated the density of states for a Permalloy-like material using the lowest branch of the magnetoexchange spectrum [12], [13]. A representative density of states profile for a 20-nm thick film with the following properties is shown in Fig. 2(a): exchange constant $A=1.0 \times 10^{-11} \mathrm{~J} / \mathrm{m}$ and $M s=8.0$ $\times 10^{5} \mathrm{~A} / \mathrm{m}$ with an applied field $\mu_{0} H==100 \mathrm{mT}$ such that the resonance is found at $9.8 \mathrm{GHz}$. The lower limit of the density of states in Fig. 2(a) corresponds to the bottom of the spin-wave manifold at 9.4 GHz, and the upper limit is an arbitrary cutoff. Fig. 3(a) and (b) shows the corresponding intensities of the new spin-wave modes coupled by the addition of these inhomogeneities with the varying strength. The effect on the original density of states due to the perturbation field is shown in Fig. 3(a) and (b). 

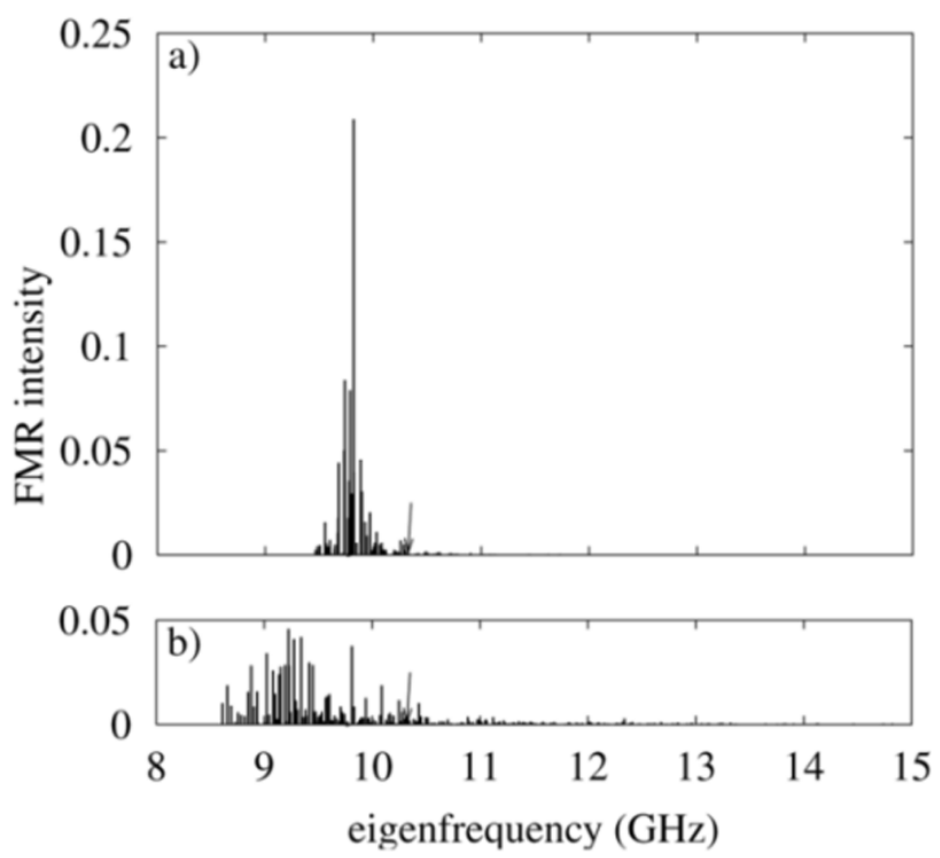

Fig. 3. Intensity of rediagonalized eigenmodes for a system with a nonuniform density of states. (a) Arms $=0.02 \mathrm{GHz}$, and (b) Arms $=0.05 \mathrm{GHz}$. The tails in the data are consistent with a Lorentzian and the peak shifts with increasing perturbation strength.

There are two effects relative to the density of states that merit discussion. First, the perturbations affect the density of states, rounding the low-frequency cutoff, and smearing the small dip in density of states at the resonance frequency validating the approach of ${ }^{[9]}$. The two-magnon model leaves the density of states unchanged.

Second, the resulting intensity spectrum in Fig. 3 is spread over a frequency range that includes significant variations in the unperturbed density of states. The spectrum in Fig. 3(b) extends well beyond the low-frequency cutoff of the density of states at $9.4 \mathrm{GHz}$ in Fig. 2(a). Because the density of states varies with frequency, the two-magnon model predicts a frequency-dependent effective damping, and this effect has been demonstrated experimentally by Patton ${ }^{[14]}$. The effect here is different in that after diagonalization, we have modes with resonant frequencies outside the initial spin-wave manifold.

Unlike the case where the initial density of states is uniform, the resulting intensity spectrum does not appear to be symmetric. The long tails that are consistent with Lorentzian line shape at high frequencies are not seen at low frequencies, and there appears to be a smeared cutoff below the bottom of the spin-wave manifold. Because there is nonzero intensity near this cutoff, this effect may appear experimentally as an asymmetric signal on the low-frequency (high field) side of the main resonance. The intensity spectra of the perturbed films also show a downward shift of the intensity maximum that is qualitatively consistent with frequency shifts predicted by the two-magnon model [2], [7]. 


\section{SECTION IV.}

\section{Conclusion}

With a rediagonalization of the fully perturbed Hamiltonian we are able to find a new set of eigenmodes. The rediagonalization allows us to study the two-magnon approximation for weak perturbations. We find for weak perturbations the rediagonalization creates a Lorentzian distribution in the FMR linewidth, is linear in the density of states and quadratic in the perturbation as predicted by the two-magnon model. The method also allows us to look beyond the two-magnon model to cases where the inhomogeneity significantly affects the density of states, or to cases where the inhomogeneously broadened spectrum is wide compared to significant variations in the density of states of the unperturbed film.

\section{References}

${ }^{1}$ E. Schlömann (Schlomann), "Ferromagnetic resonance in polycrystals", J. Phys. Rad., vol. 20, pp. 327332, 1959.

${ }^{2}$ A. M. Clogston, H. Suhl, L. R. Walker, P. W. Anderson, "Ferromagnetic resonance line width in insulating materials", J. Phys. Chem. Solids, vol. 1, pp. 129-136, 1956.

${ }^{3}$ M. Sparks, R. Loudon, C. Kittel, "Ferromagnetic relaxation. I. Theory of the relaxation of the uniform precession and the degenerate spectrum in insulators at low temperatures", Phys. Rev., vol. 122, pp. 791-803, 1961.

${ }^{4}$ D. Ter Haar, Fluctuation Relaxation and Resonance in Magnetic Systems, New York:Plenum, pp. 69, 1961.

${ }^{5}$ G. T. Rado, H. Suhl, Magnetism, New York:Academic, vol. 1, pp. 449, 1963.

${ }^{6}$ M. J. Hurben, C. E. Patton, "Theory of two magnon scattering microwave relaxation and ferromagnetic resonance linewidth in magnetic thin films", J. Appl. Phys., vol. 83, pp. 43444365, 1998.

${ }^{7}$ R. Arias, D. L. Mills, "Extrinsic contributions to the ferromagnetic resonance response of ultrathin films", Phys. Rev. B, vol. 60, pp. 7395-7409, 1999.

${ }^{8}$ E. Schlömann (Schlomann), "Inhomogeneous broadening of ferromagnetic resonance lines", Phys. Rev., vol. 182, pp. 632-645, 1969.

${ }^{9}$ Q. H. F. Vrehen, "Absorption and dispersion in porous and anisotropic polycrystalline ferrites at microwave frequencies", J. Appl. Phys., vol. 40, pp. 1849-1860, 1969.

${ }^{10}$ R. W. Damon, J. R. Eshbach, "Magnetostatic modes of a ferromagnetic slab", J. Phys. Chem. Solids, vol. 19, pp. 308-320, 1961.

${ }^{11}$ T. Wolfram, R. E. De Wames, "Magnetoexchange branches and spinwave resonance in conducting and insulating films: Perpendicular resonance", Phys. Rev. B, vol. 4, pp. 3125-3141, 1971.

12 B. A. Kalinikos, A. N. Slavin, "Theory of dipole-exchange spin wavespectrum for ferromagnetic films with mixed exchange boundary conditions", J. Phys. C. Solid State Phys., vol. 19, pp. 7013-7033, 1986. 
${ }^{13}$ R. D. McMichael, M. D. Stiles, P. J. Chen, W. F. Egelhoff, Jr., "Ferromagnetic resonance linewidth in thin films coupled to NiO", J. Appl. Phys., vol. 83, pp. 7039-39, 1998.

${ }^{14} \mathrm{C}$. E. Patton, "Effective linewidth due to porosity and anisotropy in polycrystalline yttrium iron garnet and CaV-substituted yttrium iron garnet at $10 \mathrm{GHz}$ ", Phys. Rev., vol. 179, pp. 352-358, 1969. 